\title{
Modeling of electron cyclotron resonance acceleration in a stationary inhomogeneous magnetic field
}

\author{
Valeri D. Dougar-Jabon and Eduardo A. Orozco \\ Universidad Industrial de Santander, A.A. 678 Bucaramanga, Colombia \\ Anatoly M. Umnov \\ Russian Friendship University, W117198 Moscow, Russia \\ (Received 27 November 2007; published 22 April 2008)
}

\begin{abstract}
In this paper, the cyclotron autoresonance acceleration of electrons in a stationary inhomogeneous magnetic field is studied. The trajectory and energy of electrons are found through a numerical solution of the relativistic Newton-Lorentz equation by a finite difference method. The electrons move along a $\mathrm{TE}_{112}$ cylinder cavity in a steady-state magnetic field whose axis coincides with the cavity axis. The magnetic field profile is such that it keeps the phase difference between the electric microwave field and the electron velocity vector within the acceleration phase band. The microwaves amplitude of $6 \mathrm{kV} / \mathrm{cm}$ is used for numerical calculations. It is shown that an electron with an initial longitudinal energy of $8 \mathrm{keV}$ can be accelerated up to $260 \mathrm{keV}$ by $2.45 \mathrm{GHz}$ microwaves at a distance of $17 \mathrm{~cm}$.
\end{abstract}

DOI: 10.1103/PhysRevSTAB.11.041302

PACS numbers: 52.20.Dq, 52.65.Cc

\section{INTRODUCTION}

The self-sustenance of the cyclotron resonance interaction between the electron and the transverse electromagnetic wave which is propagated along the homogeneous magnetic field has been known since 1962 [1,2]. This phenomenon, named as autoresonance, has come to scientists' attention because it opens some new methods to achieve high particle energies. Numerous theoretical and experimental studies are made in order to find the optimal conditions for the effective autoresonance electron acceleration in the homogeneous magnetic fields [3-6]. The electron cyclotron resonance conditions can also be maintained in time through the GYRAC mechanism [7], according to which the electrons are found captured in the middle plane of a mirror magnetic trap and accelerated to very high energies by microwaves due to a pulse rise of the magnetic field level. The magnetic field level rises with a rate required for a precise compensation of a relativistic deflection of the electron cyclotron movement phase on the wave phase. An acceleration of electrons by microwaves to high energies is also found in numerical experiments on plasma dynamics in an electron cyclotron resonance (ECR) minimum-B trap [8]. Experiments on the electron cyclotron plasma heating by pulse microwaves in an adiabatic mirror magnetic field show that a fraction of plasma electrons can achieve relativistic energies [9]. In these simulations and experiments, the high energy electron groups are attributed to the autoresonance phenomenon. Unlike the GIRAC non-steady-state magnetic field, the magnetic fields of the minimum-B trap and of the mirror trap, where an autoresonance is supposedly observed, are stationary and inhomogeneous. If the phenomenon of automaintenance of the ECR conditions in the stationary inhomogeneous magnetic field is a reality, it may be named as spatial autoresonance. In this paper, the spatial autoresonant acceleration hypothesis is subjected to a direct numerical study in the one-particle approximation.

\section{SIMULATION MODEL}

It is well known that the electron autoresonance regime consists in the constant maintenance of the equality between the frequency $\omega$ of the stationary electromagnetic wave and the cyclotron frequency given by the relation $\Omega_{C}=e B / m_{0} \gamma$, where $e$ and $m_{0}$ are the charge and the rest mass of the electron, $B$ is the magnetic field, and $\gamma$ is the relativistic factor. In the process of the resonance interaction, the relation $\Omega_{C}=\omega$ can be satisfied through an increase in time of the magnetic field level at a rate required to compensate the increase of the relativistic electron mass [1-7]. In case of the spatial autoresonance, the magnetic field is a function of the coordinates, and not of time. Thus, the electron cyclotron frequency is given by

$$
\Omega_{C}(\vec{r})=e B(\vec{r}) / m_{0} \gamma(\vec{r}),
$$

where $\gamma(\vec{r})=\left\{1-\left[v^{2}(\vec{r}) / c^{2}\right]\right\}^{-1 / 2}$ and the $z$-axis is aligned with the magnetic field axis. The spatial autoresonance acceleration is different from the other autoresonance types because in the inhomogeneous magnetic field there appears a diamagnetic force which acts in the direction opposite to the magnetic field gradient and impedes the advance of the electrons into a higher magnetic field. The spatial autoresonance acceleration comes to a stop when the longitudinal electron energy decays to zero due to the work of the diamagnetic force or when the synchrotron radiation power becomes equal to the microwave power absorbed by electrons. In the present numerical experiments, the synchrotron radiation is not taken into account because the electron energies are not expected to 


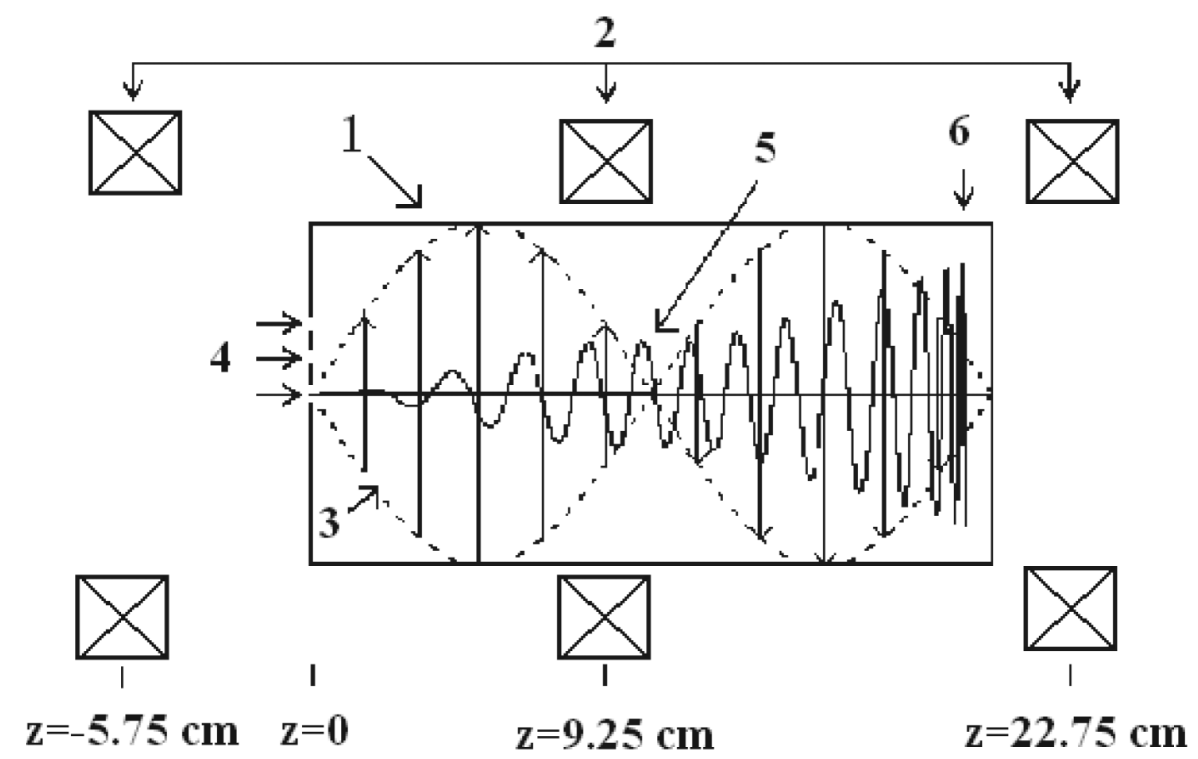

FIG. 1. A physical model scheme: 1-cavity, 2-magnetic coils, 3-electric field profile, 4-electron injection points, 5electron trajectory, 6-stop plane.

be high enough. The physical simulation scheme for the spatial autoresonance acceleration of electrons is shown in Fig. 1.

The cylindrical $\mathrm{TE}_{112}$ cavity is excited by $2.45 \mathrm{GHz}$ microwaves at an amplitude of $6 \mathrm{kV} / \mathrm{cm}$. The cavity radius and length are 4.54 and $20 \mathrm{~cm}$, respectively. The right-hand polarized microwave field is approximated by the expression: $\vec{E}=E_{0}(\cos \omega t \hat{i}+\sin \omega t \hat{j}) \sin (2 \pi z / d)$. The cavity radius was chosen larger than the expected maximum Larmor radius. The static magnetic field is generated by a system of 3 coil currents. The components $B_{r}(r, z)$ and $B_{z}(r, z)$ are calculated on a rectangular grid whose longitudinal cross section $(r, z)$ is a rectangular of $26 \times 81$ cells. The azimuth angle $\theta$ is introduced indirectly through the $(x, y)$ coordinates determined in the cross section planes: $B_{x}=(x / r) B_{r}$ and $B_{y}=(y / r) B_{r}$. The magnetic field values in the particle position points are calculated through the bilinear interpolation method. The geometric parameters and positions of the coils are presented in Fig. 1 and in Table I, where $R_{i}, R_{e}, L_{b}$, and $z$ are the internal radius, the external radius, the width of each coil, and the positions of the coils, respectively, and $j$ is the coil current density. The two-dimensional profile of the magnetic field in units of $B_{0}=m_{0} c \omega / e=875 \mathrm{G}$ is shown in Fig. 2.

The electron movement is described by the NewtonLorentz equation which in its dimensionless form is

TABLE I. Magnetic coil system parameters.

\begin{tabular}{lccccr}
\hline \hline Coils & $R_{i}(\mathrm{~cm})$ & $R_{e}(\mathrm{~cm})$ & $L_{b}(\mathrm{~cm})$ & $j\left(\mathrm{~A} / \mathrm{mm}^{2}\right)$ & $z(\mathrm{~cm})$ \\
\hline 1 & 6.0 & 20.0 & 6.0 & 1.48 & -5.75 \\
2 & 6.0 & 20.0 & 7.5 & 1.23 & 9.25 \\
3 & 6.0 & 20.0 & 10.0 & 1.29 & 22.75 \\
\hline \hline
\end{tabular}

$$
\frac{d \vec{u}}{d \tau}=-\left(\vec{g}_{0}+\frac{\vec{u}}{\gamma} \times \vec{b}\right)
$$

where $\vec{u}=\vec{p} / m_{0} c$-electron impulse; $\vec{g}_{0}=e \vec{E} / m_{0} c \omega-$ microwave electric field; $\vec{b}=\vec{B} / B_{0}$-magnetic field; $\tau=\omega t$-time; and $\gamma=\left(1+u^{2}\right)^{1 / 2}$-relativistic factor. The Eq. (2) in the finite difference form can be written as

$$
\frac{\vec{u}^{n+1 / 2}-\vec{u}^{n-1 / 2}}{\Delta \tau}=-\left(\vec{g}^{n}+\frac{\vec{u}^{n+1 / 2}+\vec{u}^{n-1 / 2}}{2 \gamma^{n}} \times \vec{b}^{n}\right) .
$$

Equation (3) is solved numerically using the Boris scheme where the coordinates of the particles are taken

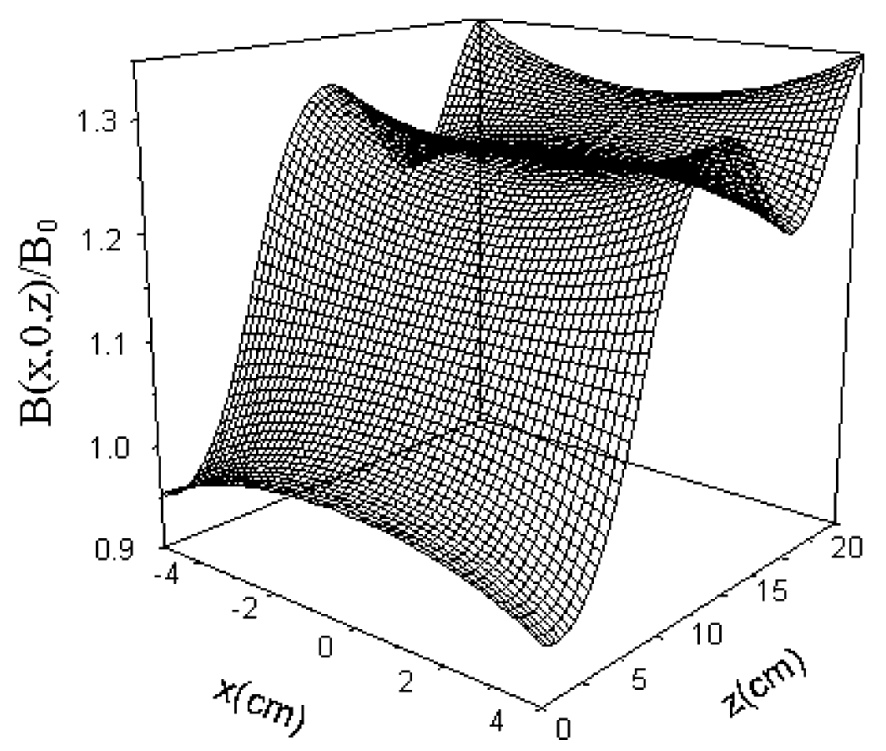

FIG. 2. The profile of the magnetic field in the $y=0$ plane. 
as follows:

$$
\vec{x}^{n+1}=\vec{x}^{n}+\vec{u}^{n+1 / 2} \Delta \tau / \gamma^{n+1 / 2},
$$

where $\gamma^{n+1 / 2}=\left[1+\left(\vec{u}^{n+1 / 2}\right)^{2}\right]^{1 / 2}$. All the length values are expressed in units of the relativistic Larmor radius $\rho_{L 0}=c / \omega$.

\section{RESULTS AND DISCUSSIONS}

For the numerical experiments, the electrons are injected into the cavity along the $z$-axis through three radial points that are $r_{0}=0,0.4$, and $1.5 \mathrm{~cm}$ (see Fig. 1). The initial longitudinal energy of the electrons is taken equal to 8,10 , and $12 \mathrm{keV}$ but the initial transverse energy is chosen nil. Figure 3 shows the calculated spiral trajectory of an electron which is injected along the cavity axis, $r_{0}=0$, with an energy of $10 \mathrm{keV}$. The electron trajectory projected in any transverse plane is a ring whose radius is a Larmor radius determined by the electron energy and the local magnetic field value. Evolutions of the electron transverse energy are presented in Fig. 4. One can see that the electron, when injected right along the cavity axis, comes to a stop in the plane of $z=19 \mathrm{~cm}$ owing to the diamagnetic force work. In this plane, the electron achieves energy of $230 \mathrm{keV}$. As shown in Fig. 4, the greater the initial radial position, the smaller is the acceleration efficiency. For the $z=19 \mathrm{~cm}$ position, the energy of the electron injected at $r_{0}=0.4 \mathrm{~cm}$ is $216 \mathrm{keV}$, and the electron starting at $r_{0}=1.5 \mathrm{~cm}$ reaches only $196 \mathrm{keV}$. Under these conditions, the acceleration efficiency for the case of $r_{0}>0$ is found lower than for the $r_{0}=0$ case. All electrons that are injected through an orifice of $0.6 \mathrm{~cm}$ in diameter come to a stop between the

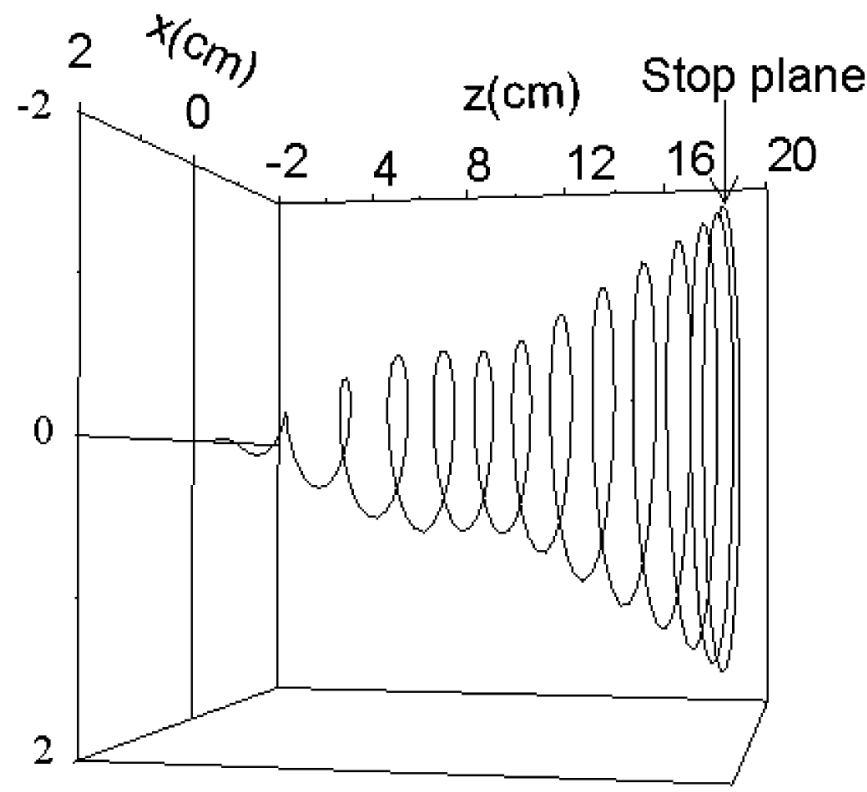

FIG. 3. The trajectory of an electron injected at the position $r_{0}=0$.

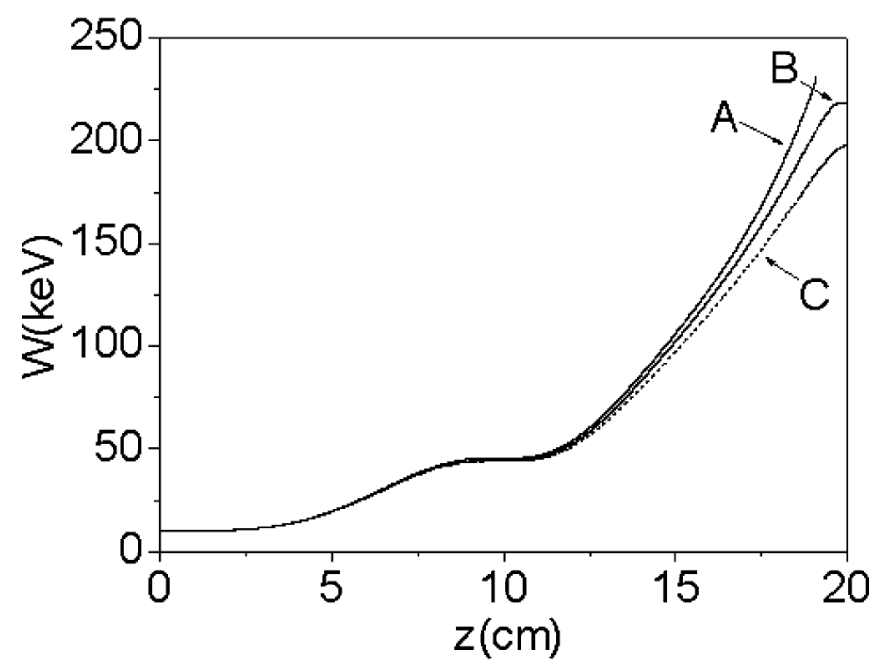

FIG. 4. The electron energy evolution for different radial injection positions: (A) $r_{0}=0.0 \mathrm{~cm}, \quad$ (B) $r_{0}=0.4 \mathrm{~cm}$, (C) $r_{0}=1.5 \mathrm{~cm}$.

positions $z=19 \mathrm{~cm}$ and $z=20 \mathrm{~cm}$. The energy dispersion of these electrons is found to be not higher than $10 \%$.

The quantitative equality between the cyclotron frequency and the microwave field frequency, being a necessary condition for the resonance acceleration, is not sufficient. The electron can absorb the electromagnetic energy only if the difference $\varphi$ between the electric field phase $\varphi_{\vec{E}}$ and the phase of the electron movement $\varphi_{\vec{V}}, \varphi=$ $\varphi_{\vec{E}}-\varphi_{\vec{V}}$, lies in a band of $(\pi / 2,3 \pi / 2)$. Figure 5 shows the evolution of the phase difference $\varphi$ when the electron advances along the $z$-direction. For the plane $z=0$, the exact resonance phase difference $\varphi=\pi$ is observed only for the electron whose initial position is $r_{0}=0$; the initial phase of other electrons differs from $\pi$ due to the radial inhomogeneity of the magnetic field. The cyclotron rota-

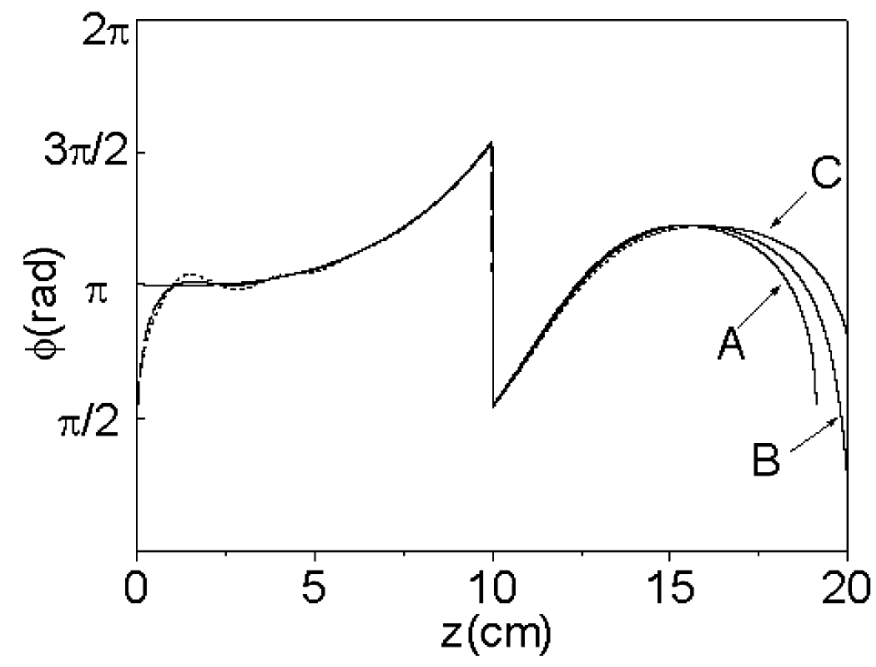

FIG. 5. Dependence of the phase difference $\varphi$ on the longitudinal coordinate $z:$ (A) $r_{0}=0.0 \mathrm{~cm}, \quad$ (B) $r_{0}=0.4 \mathrm{~cm}$, (C) $r_{0}=1.5 \mathrm{~cm}$. 
tions lead to a rapid agreement between the particle velocity phase and the electric field phase if the longitudinal velocity is relatively small. In our case, it occurs at a distance of $1.0 \mathrm{~cm}$ from the plane $z=0$ (see Fig. 5). All the electrons in the region $1<z<4 \mathrm{~cm}$ are in the nearexact resonance conditions, $\varphi \approx \pi$; however, their transverse energies do not change considerably because the $\mathrm{TE}_{112}$ electric field in this region is still small. Then, in an interval between $z=5 \mathrm{~cm}$ and $z=8 \mathrm{~cm}$, a gradual increase in the transverse electron energy is observed. In spite of the fact that the electrons remain in a favorable phase band, the energy increase rate is not high because the microwave field in this region decreases (see Fig. 1) and the phase $\varphi$ goes away from the exact resonance value (see Fig. 5). The electric field falls to zero in the point $z=$ $10 \mathrm{~cm}$ where the phase difference for all particles jumps from $\varphi \approx 4 \pi / 3$ to $2 \pi / 3$. This jump does not remove the phase $\varphi$ from the acceleration band. While the particles continue to penetrate into the cavity the phase difference approaches the exact resonance value (see Fig. 5). So, in the vicinity of $z=15 \mathrm{~cm}$, where the microwave field achieves its maximum, the electrons absorb effectively the microwave power, which results in the fact that the energy increase rate is found higher in the second half of the $\mathrm{TE}_{112}$ standing wave. For the electron injected at $r_{0}=$ $0.4 \mathrm{~cm}$, the energy decrease near the lateral cavity wall is caused by an unfavorable phase difference. As displayed in Fig. 6, the energy which is gained by the electrons injected along the cavity axis is dependent on the initial longitudinal energy. The electron with an initial longitudinal energy of $8 \mathrm{keV}$ is accelerated up to $265 \mathrm{keV}$. It happens when the electron reaches the plane $z=17 \mathrm{~cm}$ where its longitudinal velocity decays to zero. The electron trajectory in this plane makes a ring with a radius of $1.75 \mathrm{~cm}$. The electron with the initial energy of $10 \mathrm{keV}$ achieves a smaller transverse energy which is $230 \mathrm{keV}$ and consequently penetrates

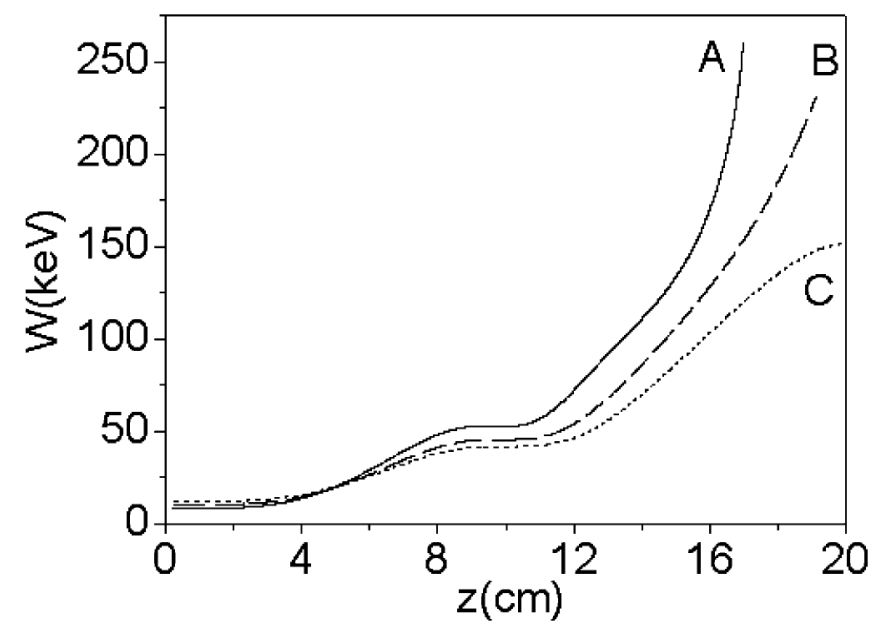

FIG. 6. Spatial evolutions of the electron energy for different initial longitudinal energies: (A) $W_{i}=8 \mathrm{keV}$, (B) $W_{i}=10 \mathrm{keV}$, (C) $W_{i}=12 \mathrm{keV}$.

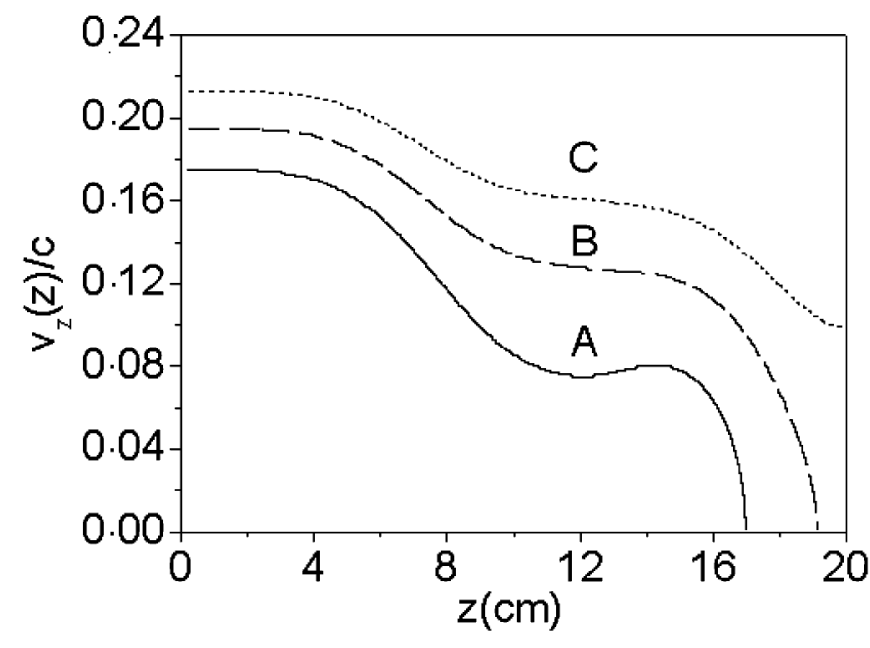

FIG. 7. Dependence of the longitudinal velocity on the longitudinal position: (A) $W_{i}=8 \mathrm{keV}$, (B) $W_{i}=10 \mathrm{keV}$, (C) $W_{i}=12 \mathrm{keV}$.

deeper into the cavity as compared with the abovementioned case of $8 \mathrm{keV}$. The longitudinal movement of the electron whose initial energy is $12 \mathrm{keV}$ does not stop inside the cavity but falls on the inner lateral cavity wall (see Fig. 6). The maximum transverse energy of this electron is only $150 \mathrm{keV}$.

Figure 7 shows the electron longitudinal velocity evolution for different initial energies; all the other parameters remain unchanged. A local decrease of the magnetic field value in the range of (12-14) $\mathrm{cm}$ provokes a slight increase in the longitudinal velocity which is detectable only for the minimum initial energy case $W_{i}=8 \mathrm{keV}$ (see Fig. 2).

Figure 8 shows the variation of the Larmor radius as the electrons advance into the cavity. One can see that the Larmor radius of the electron whose guiding center moves along the magnetic field axis is changed smoothly. In the case of $r_{0}>0$, oscillations of the electron Larmor radius

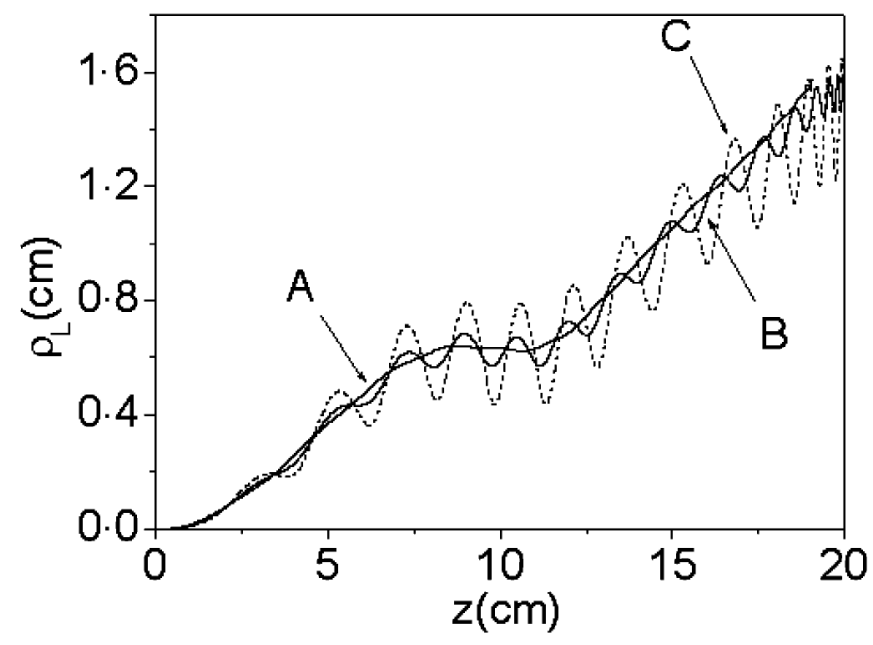

FIG. 8. Larmor radius versus the electron longitudinal positions: (A) $r_{0}=0.0 \mathrm{~cm}$, (B) $r_{0}=0.4 \mathrm{~cm}$, (C) $r_{0}=1.5 \mathrm{~cm}$. 
are observed. They are bound to arise because the magnetic field is radially inhomogeneous and its value is varied periodically as the electron follows its spiral trajectory. The higher initial radial position results in a greater oscillation amplitude.

\section{CONCLUSIONS}

The simulations discussed in the present paper evidence that the electrons can be accelerated by microwaves in an inhomogeneous magnetic field through the automaintenance of the cyclotron resonance interaction. There is no doubt that the spatial autoresonance mechanism can accelerate electrons up to energies of the order of hundreds of kilo-electron-volts and possibly Mega-electron-volts at acceptable equipment characteristics.

\section{ACKNOWLEDGMENTS}

This work was supported by PRODEIN-2007 program at the Universidad Industrial de Santander and Colciencias under Contract No RC 199-2003.

[1] A. A. Kolomenskii and A. N. Levedev, Dokl. Akad. Nauk USSR 145, 1251 (1962) [Sov. Phys. Dokl. 7, 492 (1962)].

[2] V. Ya. Davydovskii, Zh. Eksp. Teor. Fiz. 43, 886 (1962) [Sov Phys. JETP 16, 629 (1963)].

[3] H. R. Jory and A. W. Trivelpiece, J. Appl. Phys. 39, 3053 (1968).

[4] C. Roberts and S. Buchsbaum, Phys. Rev. 135, A381 (1964).

[5] C. Chen, Phys. Fluids B 3, 2933 (1991).

[6] A. P. Ishkov, Izv. Vusov, Fisica. 2, 176 (1970) (in Russian).

[7] R. Geller and K. S. Golovanivsky, Nucl. Instrum. Methods Phys. Res., Sect. B 68, 479 (1999).

[8] V. D. Dougar-Jabon, A. M. Umnov, and D. Suescun Díaz, Rev. Sci. Instrum. 73, 629 (2002).

[9] K.F. Sergeichev, D. M. Karfidov, and N. A. Lukina, Plasma Phys. Rep. 33, 455 (2007). 